\title{
Control estratégico de los agentes de aduanas del estado Zulia (Venezuela)*
}

\author{
Morán Quintero, Joselyn** \\ Ferrer, María Alejandra***
}

\section{Resumen}

El control estratégico permite establecer reglas, métodos y herramientas para medir y analizar la congruencia, el avance y la efectividad en el logro de los objetivos. Así, el objetivo de este trabajo es analizar el control estratégico de los agentes de aduanas del estado Zulia (Venezuela). Sustentada en los aportes teóricos de Kaplan y Norton (1997 y 2009), Castaño (1998), Münch y García (2005), Mintzberg et al (1997), Quintero et al (2010), Bohórquez (2011), Ferrer y Pelekais (2004), Nava et al (2008), entre otros, esta investigación es de tipo descriptiva, con diseño no experimental, transeccional y de campo. Para la recolección de datos fue aplicado un cuestionario auto-administrado a personal clave de 39 agentes de aduanas del estado Zulia. Los resultados evidencian que las empresas objeto de estudio aplican los siguientes controles estratégicos: a) medidas de desempeño global; b) estándares de comparación de los resultados reales; c) controles de calidad de servicio; d) análisis de los resultados financieros; e) informes contables; y f) retroalimentación oral y escrita. Se concluye que los agentes de aduanas consideran diferentes controles estratégicos, siendo necesarios su articulación así como el desarrollo de medidas no financieras, que sumadas a los indicadores existentes, han de proporcionar una visión global del desempeño.

Palabras clave: Control estratégico, agentes de aduanas, Venezuela.

\section{Recibido: 22-02-14 Aceptado: 08-01-15}

* Este trabajo constituye un avance del Proyecto de Investigación (CH-0064-13) "Información para la gestión privada", financiado por el Consejo de Desarrollo Científico y Humanístico (CONDES-LUZ), y adscrito al Centro de Estudios de la Empresa (CEE) de la Universidad del Zulia (LUZ).

** Licenciada en Contaduría Pública. Magíster en Gerencia de Empresas, Mención Gerencia Financiera. Docente de la Universidad del Zulia. Coordinadora de Administración en Aduanera Marítima Aérea, C.A. Acreditada por el Programa de Estímulo a la Innovación e Investigación (PEII), Nivel A-1, e-mail: joselynmoran@gmail.com

*** Licenciada en Contaduría Pública. Magíster en Gerencia de Empresas, Mención Gerencia Financiera. Doctora en Finanzas de Empresa. Docente-Investigadora de la Universidad del Zulia. Acreditada por el Programa de Estímulo a la Innovación e Investigación (PEII), Nivel B, e-mail: maferrer99@yahoo.com 


\section{Strategic Control of Customs Agents in the State of Zulia (Venezuela)}

\section{Abstract}

Strategic control permits establishing rules, methods and tools to measure and analyze congruence, advance and effectiveness in achieving objectives. Therefore, the objective of this work is to analyze the strategic control of customs agents in the State of Zulia (Venezuela). Supported by the theoretical contributions of Kaplan and Norton (1997 and 2009), Castaño (1998), Münch and García (2005), Mintzberg et al (1997), Quintero et al (2010), Bohórquez (2011), Ferrer and Pelekais (2004) and Nava et al (2008), among others, this research is of the descriptive type with a non-experimental, cross-sectional, field design. To collect data, a self-administered questionnaire was applied to key personnel of 39 customs agents in the State of Zulia. Results show that the companies under study applied the following strategic controls: a) overall performance measurements, b) comparison standards for real results, c) service quality controls, d) financial results analysis, e) accounting reports and f) oral and written feedback. Conclusions were that customs agents consider different strategic controls whose articulation is necessary as is the development of non-financial measures that, added to the existing indicators, should offer an overall vision of performance.

Key words: Strategic control, customs agents, Venezuela.

\section{Introducción}

El papel que desempeñan los agentes de aduanas en el comercio internacional es indudable, por cuanto son partícipes del intercambio de bienes entre dos o más países o regiones económicas. El dinamismo inherente a la actividad comercial demandó la incorporación de entes intermediarios entre las Aduanas (Auxiliares de la Administración Tributaria) y los particulares (personas naturales y/o jurídicas).

En Venezuela, la legislación cataloga a estos intermediarios como agentes de aduanas, entes que sirven de apoyo en todo lo relacionado en materia aduanera ${ }^{1}$. Su campo de actuación se circuns- cribe a determinada región del país: región Centro-Norte Costera, región de los Llanos Centrales, región Centro Occidental, región Zuliana, región de Los Andes, región del Sur, región Nor-Oriental, región Guayana y región Insular.

Ahora bien, de acuerdo con los datos estadísticos emanados por el Instituto Nacional de Estadísticas (2012), durante el período 1998-2012 el sector aduanero en Venezuela sufrió cambios a gran escala y de forma impredecible generados por diversos eventos de carácter interno y externo. Al respecto destaca el paro petrolero del año 2002, que supuso la paralización general en las operaciones portuarias y aduanales; la reactivación de las actividades tardó aproximadamente cin- 
co meses, por lo que muchos agentes de aduanas cerraron sus puertas debido a la reducción de sus ingresos.

Posteriormente, el control cambiario impuesto a partir de febrero de 2003 (Asamblea Nacional de la República Bolivariana de Venezuela, 2003), causó nuevamente un impacto en las operaciones de las empresas, pues los importadores y exportadores fueron sometidos por un nuevo proceso burocrático y limitado para la adquisición de divisas. A partir de la entrada en vigencia del nuevo régimen cambiario, la solicitud de divisas para importación viene a ser el resultado de un proceso administrativo riguroso, lo que se tradujo en una reducción significativa de las operaciones aduaneras.

En términos generales, las restricciones legales y económicas impuestas a nivel nacional afectan el ritmo de las operaciones de compra y venta de bienes con otros países y, en consecuencia, limitan las operaciones de los agentes de aduanas al punto de que la Asociación de Agentes Aduanales del estado Zulia (ASADAEZ), manifestó el cese de operaciones de muchas de ellos durante el período 2003-2007 (Arcaya, 2007).

Si bien los agentes de aduanas del estado Zulia no han escapado a la disminución de los trámites de importaciones y exportaciones, aún se encuentran activas un número importante de empresas dedi- cadas a la prestación de servicios aduaneros a personas jurídicas y naturales.

Día a día estas empresas han de satisfacer las exigencias y necesidades internas, así como las particularidades o desafíos de un entorno cada vez más complejo. Para dar respuesta a éstas y, al mismo tiempo, alcanzar los objetivos propuestos, este grupo de empresas plantean una serie de estrategias dirigidas a la implantación y desarrollo de actividades enfocadas en esos objetivos, y que exigen verificar: a) si la estrategia se está implementando como se planificó; y b) si los resultados generados por esa estrategia son los esperados. En este contexto los agentes demandan métodos, herramientas y formas de trabajo que les permitan conocer y medir la congruencia, el avance y la efectividad en el logro de los objetivos y metas propuestas por la gerencia.

Si el ambiente externo e interno no es monitoreado continuamente, las desviaciones financieras y no financieras pueden conducir al fracaso de la organización cuando las mismas no se detectan y corrigen a tiempo. De allí la importancia del control estratégico, el cual se centra en el seguimiento y la evaluación del proceso de administración estratégica para asegurar el adecuado funcionamiento de la empresa. El mismo permite, no solo verificar los hechos del pasado con el propósito fundamental de identificar las desviacio-

dicas autorizadas por el Ministerio del Poder Popular de Economía, Finanzas y Banca Pública con competencia en materia de finanzas para actuar ante los órganos competentes en nombre y por cuenta de aquél que contrata sus servicios, en el trámite de una operación o actividad aduanera. 
nes relacionadas con el logro de los objetivos del plan estratégico, sino también el análisis de sus causas y efectos, para diseñar las acciones correctivas que garanticen la buena marcha hacia el futuro.

Con base en lo anterior, y para tratar de dar respuesta a la interrogante: ¿cómo es el control estratégico de las unidades objeto de estudio?, este trabajo se centra en analizar el control estratégico de los agentes de aduanas del estado Zulia, como vía para detectar, corregir y orientar los esfuerzos y recursos financieros y no financieros hacia la consecución exitosa de los objetivos establecidos por la empresa.

Esta investigación es considerada de tipo descriptiva, con diseño no experimental, de campo y transeccional. La población se determinó con base en la información suministrada por la Aduana Principal de Maracaibo. Así, en el estado Zulia, para el año 2012, se encontraban registrados, actualizados y en operación 39 agentes de aduanas. Puesto que la población se consideró finita y accesible, se acudió al censo población para la evaluación de la totalidad de las unidades de análisis objeto de estudio. Para la obtención de los datos empíricos se aplicó un cuestionario auto-administrado bajo escala tipo Likert, conformado por 11 afirmaciones de alternativas fijas y un pregunta de opción múltiple, dirigido a gerentes, administradores, contralores, socios y demás personal directivo de los agentes de aduanas del estado Zulia.

El instrumento de recolección de datos fue validado por siete (07) expertos en el área y la confiabilidad del instrumento se determinó mediante el coeficiente Alfa de Cronbach, resultando 0,889 para las afirmaciones tipo Likert y 0,880 para las alternativas de selección múltiple, lo cual indica la alta confiabilidad del instrumento. Los datos obtenidos fueron analizados mediante la aplicación de las funciones de la estadística descriptiva, distribución de frecuencias absolutas y frecuencias relativas y media aritmética, a través del SPSS (versión 15.0).

\section{Algunas generalidades acerca del control estratégico}

El control estratégico o control organizacional es considerado por Kaplan y Norton (1997), como un sistema de decisiones de la cúpula, que controla el desempeño y los resultados de la empresa en su totalidad, mecanismo mediante el cual considera las informaciones de retroalimentación provenientes del ambiente externo a la empresa y del nivel intermedio. Con relación a esto último, Castaño (1998) señala que la atención del control estratégico es tanto sobre aspectos internos como externos; se enfoca tanto en el macro y microambiente como en el ámbito interno de la organización, pues estos elementos no deben considerarse de manera aislada.

Para Navajo (2009), el control estratégico es un proceso en el que se mide y valora cualquier actividad con base en criterios previamente definidos, así como la corrección de las desviaciones respecto a los criterios fijados como puntos de referencia. El diseño de un sistema de control estratégico requiere de algunos elementos fundamentales (Navajo, 2009: 147): eficacia y eficiencia, continuidad, seguridad y objetividad, adecuado y aceptado por los miembros, oportunidad y enfoque sobre puntos estratégicos. 
Como función de gestión, el control permite realizar evaluaciones de los resultados $y$ tomar las medidas necesarias para minimizar las ineficiencias (Nava et al, 2008). Münch y García (2005), indican que los controles pueden estar situados en el nivel institucional, en el nivel intermedio o en el nivel operacional, o incluso diseminados en todos ellos, según las características de la empresa. El Cuadro 1, muestra los tipos de control estratégico, así como los elementos de cada uno de ellos (Castaño, 1998; Kaplan y Norton, 1997).

De acuerdo con los planteamientos de Kaplan y Norton (1997), Münch y García (2005) y Bohórquez (2011) el nivel institucional utiliza sistemas de control para medir el desempeño global de la empresa. En algunos casos, se requieren sistemas de control para evaluar una o todas las unidades, departamentos o divisiones de la organización o incluso de ciertos proyectos prioritarios.

Un sistema de control efectivo permite medir constantemente los resultados obtenidos; así, cuando éstos no son favorables es posible aplicar los correctivos necesarios para lograr el plan estratégico propuesto. El uso y la combinación de diferentes tipos de herramientas de control permiten detectar desviaciones $y$ así como el origen de las mismas indistintamente del nivel organizacional en el cual se presenten, coadyuvando de esta forma con la consecución de los objetivos estratégicos.

Generalmente los gerentes suponen que los controles globales en una or-

\section{Cuadro 1}

Tipos y Herramientas de Control Estratégico

\section{Tipos de control estratégico}

Desempeño global

Control de ganancias y pérdidas

Informes contables

Análisis de retorno de la inversión

Cuadro de mando integral

\section{Herramientas de control}

- Establecimiento de estándares

- Medición de la efectividad de las técnicas de mercadeo (marketing)

- Medición de la calidad del producto/servicio

- Detección de variaciones

- Control interno

- Medidas correctivas

- Retroalimentación

- Uso de la tasa interna de retorno para el análisis del retorno de la inversión

- Definición de indiciadores para evaluar la perspectiva financiera

- Definición de indiciadores para evaluar la perspectiva del cliente

- Definición de indiciadores para evaluar la perspectiva sobre procesos internos

- Definición de indiciadores para evaluar la perspectiva de formación y crecimiento 
ganización son sólo de carácter financiero (Kaplan y Norton, 1997). Este enfoque netamente financiero del control, supone una visión parcial de la realidad empresarial. Para Kaplan y Norton (1997) y Mintzberg et al (1997: 291), el control estratégico debe evaluar tanto el comportamiento como el desempeño de la organización en términos globales. Solo de esta manera será posible determinar la efectividad de las estrategias, tanto en términos financieros como no financieros. Aunque la existencia de los negocios sea justificada en términos de generación de utilidades y el dinero, y éste sea cada vez más un recurso escaso y costoso, la evaluación de la eficiencia de una empresa ${ }^{2}$ no siempre es completa y adecuada cuando se mide solamente en unidades monetarias. A pesar de que en todas las organizaciones, el control del desempeño global es predominantemente financiero, algunos factores igualmente importantes no pueden ser medidos mediante índices financieros.

Para medir el desempeño global de la organización deben establecerse estándares como patrón para el control de las operaciones financieras y productivas (Kaplan y Norton, 1997; Navajo, 2009). Adicionalmente, deben evaluarse aspectos relacionados con el mercadeo y la calidad del producto y/o servicio como elementos necesarios para complementar el control financiero y operacional (Kaplan y Norton, 1997).
Con relación al establecimiento de estándares, éstos son definidos por Münch y García (2005), como una unidad de medida que sirve de modelo, guía o patrón con base en la cual se efectúa el control. A su vez, Porter et al, (2006) hacen énfasis en la evaluación de los criterios fijados para la medición del desempeño; puede cometerse el error de establecerse estándares demasiado difíciles o demasiado fáciles de alcanzar por los miembros de la organización. Con el establecimiento de estándares se pretende representar el estado de ejecución deseado a partir de los objetivos definidos por la gerencia, los cuales no deben limitarse a los niveles operativos, preferentemente, deben abarcar las funciones básicas y áreas clave de éxito (Kaplan y Norton, 1997).

Ahora bien, Münch y García (2005) y Porter et al, (2006) consideran que entre los estándares a ser adoptados por las organizaciones es necesario incluir aquellos capaces de medir la efectividad o desempeño de las técnicas de mercadeo empleados para maximizar el volumen de las ventas; esto es, medidas relacionadas con la posición en el mercado, aceptación de algún producto en el mercado, y la efectividad de las técnicas mercadológicas. Desde el punto de vista de la calidad del producto y/o servicio, deben plantearse estándares relacionados con la competencia (Münch y García, 2005; Bohórquez, 2011). Por otro lado, las condiciones y parámetros para que el talento 
humano se desempeñe satisfactoriamente son factores determinantes de la calidad del producto y/o servicio. Así, mecanismos para desarrollar y capacitar al personal, medir los programas impartidos por la gerencia, y evaluar la actuación del personal, son elementos a considerar en la productividad del talento humano (Kaplan y Norton, 1997; Bohórquez, 2011).

Ahora bien, para el control del resultado financiero se acude al estado de resultados como herramienta de control. Este estado financiero ofrece una visión resumida de los resultados obtenidos por la empresa en un determinado periodo. Comparar el estado de resultado del período actual con el de periodos anteriores, permite identificar variaciones (Kaplan y Norton, 1997; Münch y García, 2005) y detectar las áreas que requieren atención por parte de la administración (Kaplan y Norton, 1997).

En este orden de ideas, es posible comparar el estado de resultados (informe contable) del período con el proyectado para dicho periodo, todo ello con el objeto de visualizar las variaciones y responsabilizar al departamento o persona por los resultados obtenidos. La detección de variaciones o desviaciones, aplicable a todas las mediciones establecidas por la organización, permite detectar las diferencias que se presentan entre lo planificado y lo ejecutado (Münch y García, 2005; Porter et al, 2006).

Muchos sistemas de control se basan en medidas de contabilidad (informes contables) como base para el cálculo de variaciones y tendencias de cifras representativas del desempeño organizacional (Harrison y Caron, 2009). Los informes contables resumen los principales hechos de la empresa en términos de volumen de ventas, volumen de producción, gastos, costos, utilidades, utilización del capital, retorno sobre la inversión, entre otros (Kaplan y Norton, 1997).

Considerando que los informes o reportes financieros pueden ser manipulados, es fundamental aplicar otros controles inherentes, como lo son el control interno, las medidas correctivas, y la retroalimentación.

Para Muñoz (2002), el control interno nace de la necesidad de evaluar la efectividad y la confiabilidad en la protección de los bienes de una organización y de controlar el desarrollo de sus actividades y resultados financieros. De manera que el control interno también parte de los objetivos institucionales de la empresa con el propósito de medir el cumplimiento adecuado de todas sus funciones para mantener una administración eficiente.

Coopers y Lybrand (1997:107), consideran al control interno como una garantía de la empresa para evitar los fallos; su uso está orientado a la consecución de los objetivos estratégicos. En este sentido, la gerencia en reiteradas ocasiones lo concibe como una cura a todos los males de la organización, pero tal posición es equivocada. El control interno se implementa en diferentes niveles de la organización para dar respuesta a distintos objetivos. Con ello se pretende resguardar la integridad de los bienes de la organización, así como el logro de las metas propuestas.

Para Münch y García (2005), la utilidad concreta y tangible del control está en las medidas correctivas para integrar las desviaciones en relación con los estándares. Una vez establecidos los co- 
rrectivos necesarios debe verificarse que las mismas no sigan presentándose. Quintero et al, (2010), consideran las medidas correctivas como indicador valioso de control; según el planteamiento de Chiavenato (2001), la evaluación de los resultados debe detectar los errores así como generar retroalimentación necesaria para evitar que vuelvan a presentarse.

El establecimiento de medidas correctivas da lugar a la retroalimentación. La información obtenida a través de ella permite ajustar el sistema administrativo en el transcurso del tiempo. Así, la calidad de la información es determinante en el grado y rapidez de la retroalimentación del sistema (Münch y García, 2005).

Otra herramienta del control estratégico es el análisis del retorno de la inversión. Esta técnica de control global es utilizada para medir el éxito absoluto o relativo de una empresa o de una unidad departamental, en razón de las ganancias con respecto a la inversión de capital. La Tasa Interna de Retorno (TIR) iguala el valor de los ingresos de un proyecto al valor presente de los egresos; es decir, es la tasa de interés con la que el valor presente neto de un proyecto es igual a cero (Álvarez, 2005). Según Gitman (2003), la TIR mide la rentabilidad producto de la reinversión de los flujos netos de efectivo dentro de la operación propia del negocio y se expresa en porcentaje. Mediante la tasa interna de retorno, la empresa evalúa sus diferentes líneas de productos y verifica dónde está siendo empleado el capital con mayor eficiencia, además de poder aplicar equilibradamente el capital para alcanzar una mayor ganancia global.
Finalmente, Kaplan y Norton (2009), señalan que el Cuadro de Mando Integral (CMI) es un sistema de control estratégico de gestión dirigido a la construcción de indicadores de desempeño clave, adecuados a la naturaleza de la empresa, al tipo de información de gestión estratégica demandada por los gerentes, y a los roles desempeñados por ellos. De acuerdo con Kaplan y Norton (1997; 2009); y Ferrer y Pelekais (2004), el CMI mide los procesos y la actuación de una empresa desde cuatro perspectivas: financieras, clientes, procesos internos, y formación y crecimiento.

La perspectiva financiera incluye los objetivos financieros de una empresa junto con los indicadores a utilizar para medir su consecución; tales objetivos financieros suelen relacionarse con la rentabilidad (Carrión, 2007). La perspectiva del cliente es un reflejo del mercado en el cual se está compitiendo, el cliente hacia el cual se dirige el servicio o producto, y brinda información importante para generar, adquirir, retener y satisfacer a los clientes, obtener cuota de mercado, y rentabilidad (Martínez y Milla, 2012). La perspectiva sobre procesos internos se refiere a los procesos que dan vida a la empresa y en los que se debe ser excelente para alcanzar los objetivos; es decir, desde la perspectiva de los procesos internos se identifica el estado de la cadena de valor por medio del análisis de los diferentes procesos (Nieto, 2006). Finalmente, la perspectiva de formación y crecimiento permite identificar la infraestructura (talento humano, sistemas y clima organizacional) necesaria para crear valor a largo plazo para alcanzar los objetivos 
de las perspectivas anteriores (Horngren et al, 2007).

Es importante que los indicadores definidos para cada una de las perspectivas descritas en el párrafo anterior no solo controlen la actividad pasada; los indicadores deben reflejar los resultados muy puntuales de los objetivos, pero también deberán informar sobre el avance para alcanzar esos objetivos.

\section{El control estratégico en los agentes de aduanas}

Partiendo de la premisa de que el éxito de los objetivos del plan estratégico se logra mediante la aplicación de diferentes herramientas (Kaplan y Norton, 2009), a continuación se describen los elementos del control estratégico de los agentes de aduanas del estado Zulia: desempeño global, control de ganancias y pérdidas, informes contables, análisis del retorno de la inversión y cuadro de mando integral (Tabla 1).

\subsection{Desempeño global}

Como parte de los elementos del control estratégico para medir el desempeño global, un $89,8 \%$ de los agentes de aduanas utilizan estándares relacionados con los niveles de rentabilidad que desean alcanzar (Tabla 1). Ello supone un buen punto de partida para el adecuado y efectivo control estratégico por parte de las unidades de estudio. Para Castaño (1998) y Porter et al (2006), Mintzberg et al (1997) y Bohórquez (2011) toda organización debe medir su desempeño global a través de diferentes herramientas de control, entre las cuales destaca el esta- blecimiento de estándares. Así, Kaplan y Norton (1997), Münch y García (2005) y Navajo (2009) consideran que para evaluar los resultados primero se deben establecer estándares para su comparación con los resultados obtenidos, para posteriormente aplicar las medidas correctivas necesarias. El uso de esta herramienta representa una base sólida para la aplicación del control efectivo y eficiente, pues permite la adecuada medición del desempeño con relación a los objetivos estratégicos establecidos.

Por otra parte, un $89,7 \%$ de los agentes de aduanas consultados miden la eficiencia y efectividad de las técnicas de mercadeo utilizadas, a través del crecimiento de las ventas (Tabla 1). Ello evidencia la importancia de la función de mercadeo en las finanzas y la aplicación de controles que midan la efectividad de estos departamentos o de las personas involucradas, tal como lo mencionan Porter et al, (2006) y Münch y García (2005). Así, una mayor participación en el mercado, como consecuencia de las técnicas de mercadeo para la captación de nuevos clientes es evaluada con base en información financiera.

Con relación a la evaluación de la calidad del servicio prestado por las empresas encuestadas, se evidenció que un $90 \%$ de los agentes de aduanas analizan el grado de satisfacción del cliente (Tabla 1). Este indicador no sólo permite evaluar la calidad del servicio como medida del desempeño del talento humano, pues al mismo tiempo es posible verificar la ejecución satisfactoria del trabajo operativo del Departamento de Aduanas. De esta manera, se evidencia la necesidad de aplicar controles tanto a factores finan- 
Tabla 1

Herramientas del Control Estratégico utilizadas por los Agentes

de Aduanas del estado Zulia-Venezuela (Año 2012)

Elementos del control estratégico

Frecuencia

(\%)

Desempeño global

Establecimiento de estándares de rentabilidad

$89,8 \%$

Medición de la efectividad de las técnicas de mercadeo

(crecimiento de las ventas)

$89,7 \%$

Medición de la calidad del servicio prestado

(grado de satisfacción del cliente)

$90,0 \%$

Control de ganancias y pérdidas

Determinación de las variaciones para el control de ganancias y pérdidas

$84,6 \%$

Asignación de responsabilidades al departamento o área por las desviaciones

detectadas

$94,9 \%$

Informes contables

Uso de mecanismos de control interno

$100,0 \%$

Establecimiento de medidas de control interno en el nivel operativo

$97,0 \%$

Aplicación de medidas correctivas

$89,7 \%$

Retroalimentación constante en todos los niveles

$89,7 \%$

Análisis del retorno de la inversión

Uso de la tasa interna de retorno para el análisis del retorno de la inversión

$0,0 \%$

Cuadro de mando integral

Uso del cuadro de mando integral

$0,0 \%$

Definición de indicadores para cada una de las perspectivas del cuadro

de mando integral

$0,0 \%$

Fuente: Elaboración propia a partir de los resultados obtenidos de aplicación de un cuestionario a personal clave de 39 agentes de aduanas del estado Zulia (Venezuela), durante el año 2012.

cieros como no financieros (Kaplan y Norton, 1997; Bohórquez (2011); Mintzberg (1997); Münch y García, 2005). Para los agentes de aduanas, el recurso humano resulta un elemento clave para la prestación de servicio. La evaluación del mismo es crítica para este tipo de organizaciones, pues el cumplimiento de los estándares de calidad influye positivamente en los resultados financieros y mejora los beneficios económicos de los agentes. El deterioro de la calidad del servicio presta- do puede generar una disminución de los ingresos. Ante este escenario, el cliente buscará empresas alternativas que ofrezcan servicios de calidad.

\subsection{Control de ganancias y pérdidas}

Con respecto al control de ganancias y pérdidas, se evidenció que un $84,6 \%$ de los agentes de aduanas determina e informa oportunamente sobre las variaciones en el resultado financiero de 
sus operaciones. Adicionalmente, un $94,9 \%$ identifica el departamento o área responsable de las desviaciones detectadas, a fin de aplicar las debidas correcciones (Tabla 1).

Así, los estados financieros, tanto históricos como proyectados, constituyen el punto de partida para que la empresa puede detectar y corregir a tiempo aquellas desviaciones capaces de comprometer su buen funcionamiento así como su permanencia en el tiempo (Kaplan y Norton, 1997; Porter et al, 2006; Harrison y Caron, 2009).

Toda ente económico debe evaluar oportunamente sus resultados (utilidades o pérdidas), pues de ello depende su permanencia; pérdidas significativas y recurrentes podrían conducir a la descapitalización de la empresa y posteriormente el cese de sus operaciones.

Cabe destacar que cuando esta responsabilidad recae en manos de un departamento específico, las personas involucradas en dicho proceso tienden a ser más analíticos y persuasivos en cuanto la determinación de los resultados así como las causas que los originaron.

\subsection{Informes contables}

Los informes contables muestran el resumen de los datos más relevantes del desempeño organizacional y comunican el estatus de la entidad con relación al logro de los objetivos relacionados con producción, inversiones, financiamiento, ingresos y egresos. Sin embargo, es importante aplicar medidas de control para corroborar la confiabilidad de los mismos, pues como se mencionó anteriormente, la información presentada en dichos informes puede ser manipulada.

De allí que un $100 \%$ de los agentes de aduanas empleen mecanismos de control interno para garantizar la fiabilidad de la información resumida en los estados financieros (Tabla 1). Con ello se pretende prevenir las pérdidas producto de errores y fraudes por parte de empleados con acceso a recursos financieros, o por la toma de decisiones erróneas basadas en información no confiable (Kaplan y Norton, 1997; Münch y García, 2005; Harrison y Caron, 2009). Para asegurar la efectividad de las medidas de control interno, éstas deben implantarse en todos los niveles organizacionales. Sin embargo, un $97,0 \%$ de los mecanismos aplicados por los agentes de aduanas se concentran en el nivel operativo (Tabla 1). Esto último compromete la efectividad del control interno, haciéndolo vulnerable a errores $y$ fraudes producto de la ausencia de controles en el nivel intermedio y en el institucional (Münch y García, 2005; Kaplan y Norton, 1997; Muñoz, 2002; Coopers y Lybrand, 1997).

Gracias a las ventajas del control estratégico, se pueden aplicar acciones correctivas con el objeto de reorientar las distensiones al curso normal o efectivo de operación. Para que esto sea posible, es indispensable la consignación oportuna de los informes desempeño para la aplicación de los correctivos necesarios.

En el caso de los agentes de aduana, un $89,8 \%$ de las unidades informantes consultadas considera que los informes de desempeño son entregados oportunamente a la gerencia (Tabla 1); ello hace posible emprender oportuna- 
mente las acciones correctivas donde amerite. Así, la entrega a tiempo de informes a la gerencia permite ajustar los planes y estrategias implantadas y verificar los resultados de las medidas correctivas tomadas (Münch y García, 2005; Kaplan y Norton, 1997; Quintero et al, 2010).

Como herramienta de control, los informes contables permiten la retroalimentación de los procesos llevados a cabo en una empresa. Para mejorar la calidad de la información y los procesos internos, un $89,7 \%$ de los agentes de aduanas mantienen una retroalimentación constante hacia todos los niveles organizaciones (Tabla 1).

Una visión global del desempeño organizacional, comunicada en forma verbal o escrita, y considerando todos los niveles departamentales (institucional, intermedio, y operacional), redunda en una mayor calidad de los producto y/o servicios (Münch y García, 2005; Kaplan y Norton, 1997). Igualmente, Quintero et al, (2010) y Chiavenato (2001), concuerdan en que la retroalimentación no solo permite detectar y corregir desviaciones, sino también su prevención.

\subsection{Análisis del retorno de la inversión}

A pesar de los beneficios del análisis del retorno de la inversión mediante la TIR, se observó que un $100 \%$ de los agentes de aduanas no miden la rentabilidad de las inversiones financieras (Tabla 1). Para Gitman (2003) y Álvarez (2005), esta herramienta permite medir el éxito absoluto o relativo de una organización o de una unidad departamental, en razón de las ganancias con respecto a la inversión de capital.

Los resultados descritos pueden sustentarse en la hipótesis de que los agentes aduanales podrían utilizar otros indicadores financieros como el valor presente neto, margen de utilidad neta, rentabilidad de los activos, rentabilidad del patrimonio, entre otros, como medidas para el análisis del retorno de la inversión.

\subsection{Cuadro de mando integral}

A pesar de las ventajas del CMI (Kaplan y Norton, 2009), resalta el hecho de que un $100 \%$ de los agentes de aduanas no lo utiliza como herramienta de control. Cabe la posibilidad de que los gerentes desconozcan los beneficios o bondades de esta herramienta de control. Aunque este sector no utiliza o desconoce el CMI como un balance armónico en donde se definen todos los aspectos relacionados con el éxito o fracaso de la entidad, se observa que de forma separada o aislada si consideran algunas perspectivas señaladas en el CMI.

Desde la perspectiva financiera, los agentes de aduanas establecen controles para medir sus resultados económicos (Control de Ganancias y Pérdidas); ello conduce a la maximización de la rentabilidad como uno de los objetivos primordiales para la organización (Carrión, 2007). Desde la perspectiva del cliente, los agentes de aduanas consideran al cliente como un factor clave dado que los mismos aplican técnicas de mercado y controles sobre la calidad del servicio prestado, coincidiendo de esta manera con lo señalado por Martínez y Milla (2012). 
No obstante, se evidencia que la perspectiva sobre procesos internos y la perspectiva de formación y crecimiento, perspectivas clave del cuadro de mando integral, no son definidas por los agentes de aduanas. Ello no permite la medición global del desempeño de la organización mediante el control de todas sus partes, tal como lo plantean Ferrer y Pelekais (2004). Si bien el CMI involucra necesariamente la interrelación de las cuatro perspectivas definidas por Kaplan y Norton (1997), las mismas deben acoplarse como un todo para lograr los objetivos de la planificación estratégica.

Al evidenciarse que los agentes de aduanas de forma aislada controlan solo dos perspectivas (financiera y cliente), se generan debilidades en el sistema. Lo anterior puede conducir al fortalecimiento y permanencia de desviaciones, las cuales sin duda afectarían el éxito de la planificación estratégica establecida por la organización.

\section{Conclusiones}

En los agentes de aduanas, así como en cualquier organización, el control de gestión acude a diferentes herramientas para dar seguimiento a la estrategia planteada por la gerencia. Las mismas combinan información de carácter financiero y no financiero lo cual permite una visión global del desempeño. Al respecto, las unidades objeto de estudio acuden al establecimiento de estándares como guías para el análisis de los resultados financieros, así como a la medición de la efectividad de las técnicas de mercadeo y de la calidad del servicio prestado. La primera es evaluada a través del crecimiento de las ventas y la segunda toma como referencia el grado de satisfacción del cliente.

El uso proporcional de estos mecanismos por parte de las organizaciones resalta la dimensión estratégica y global del personal directivo de los agentes aduanales ya que los mismos utilizan el control de gestión como un medio para asegurar el cumplimento efectivo de las metas propuestas. Al mismo tiempo, permite reconducir los lineamientos y las acciones por parte del personal en caso de que los resultados se desvíen de lo esperado.

Resulta pertinente analizar la capacidad de cada una de las herramientas de control utilizadas por las empresas del sector para evaluar apropiadamente los factores clave de éxito. Así, es necesario cuestionar aspectos como: ¿los estándares establecidos son adecuados?, ¿el crecimiento de las ventas miden de manera efectiva las técnicas de mercadeo?, ¿el grado de satisfacción del cliente está siempre asociado con la calidad del servicio, u obedece al trato recibido por parte del personal de la empresa?

Considerando que el ambiente tanto interno como externo es altamente dinámico y cambiante, es necesario el análisis y evaluación de la pertinencia de cada uno de los elementos del control de gestión. Más allá del uso de dichas herramientas, la revisión continúa de los fundamentos que sirven de base para su diseño e implantación constituyen la clave para el éxito.

Es importante resaltar que el uso de control permite a las empresas de este sector la corrección de las desviaciones entre lo planificado y lo ejecutado con mi- 
ras a alcanzar los objetivos de manera exitosa. Para la identificación de las variaciones los agentes de aduanas toman como referencia los resultados obtenidos en períodos anteriores $\mathrm{y} / \mathrm{o}$ los presupuestados para el período objeto de análisis. La determinación de las variaciones permite, no solo la aplicación de acciones correctivas, sino también la identificación de las personas o departamentos responsables de dichas desviaciones. Es evidente que los agentes de aduanas buscan no sólo identificar problemas existentes sino también prevenirlos; la detección de discrepancias permite aplicar los ajustes necesarios para lograr las metas deseadas, ya sea el incremento la rentabilidad, la reducción de costos y/o la maximización del valor de la organización.

Las herramientas para el control estratégico utilizadas por los agentes de aduanas se apoyan en mecanismos como el control interno. Sin embargo, éste es implantado en mayor proporción a nivel operativo. El carácter estratégico del control interno supone su implementación en todas las fases y departamentos del ente económico. Si éste se centra en un nivel organizacional, limita los beneficios derivados del mismo.

El talento humano es susceptible de cometer actos que, de manera voluntaria o involuntaria, atentan contra el cumplimiento del plan estratégico. De allí la importancia de aplicar mecanismos de control interno en todos los niveles organizacionales. En la pirámide jerárquica de una empresa es posible identificar las diferentes líneas de mandos, así como los encargados de la formulación de los objetivos, metas, normas y directrices a cumplir por parte de los niveles inferiores. Así, la verificación del cumplimiento de las normas de control interno permite evitar desviaciones con relación a lo planificado, al tiempo que contribuye al logro de los objetivos organizacionales.

De las herramientas aplicadas para el control estratégico, resalta que los agentes de aduanas del estado Zulia no utilizan el análisis de retorno de la inversión, pues no es frecuente la puesta en marcha de proyectos de inversión. Esto puede obedecer a la naturaleza de la actividad económica del sector, así como la situación económica-política del país. Por otra parte, una de las desventajas de la TIR es que su cálculo no incorpora los efectos de la inflación; ello afecta la eficiencia del indicador considerando los elevados índices inflacionarios experimentados en el país en los últimos años.

Entre los resultados obtenidos también destaca que los agentes de aduanas no utilizan el cuadro de mando integral como técnica para evaluar su desempeño. Si bien, el uso de las herramientas antes descritas facilita el control estratégico de las unidades objeto de estudio, la integración de la información financiera y no financiera bajo el esquema del cuadro de mando integral facilitaría el proceso de toma de decisiones por parte de los gerentes de la empresa. Sin embargo, se observa el uso de medidas financieras y no financieras para evaluar las perspectivas financieras y del cliente, dos de las perspectivas consideradas en el CMI. Sin embargo, no son suficientes. El desarrollo de indicadores para evaluar la perspectiva sobre procesos internos y la de formación y crecimiento es vital para que estas empresas, no solo permanezcan en el mercado de manera competitiva, 
sino también para el monitoreo del logro de los objetivos organizacionales.

Considerando que las empresas del sector se dedican a la prestación de servicios, el recurso humano representa un factor clave. La falta de formación, capacitación y estímulos, ya sea de carácter cualitativo o cuantitativo, pueden suponer en el largo plazo deficiencias en la calidad del servicio, y en consecuencia clientes insatisfechos. La situación descrita podría impactar la perspectiva financiera al generar una disminución en los niveles de ingresos. De allí, la importancia de la construcción de un balance para recopilar todos los aspectos que, directa o indirectamente, afectan los objetivos estratégicos.

Finalmente, en función del entorno altamente cambiante en el que se desenvuelven los agentes de aduanas del estado Zulia, la aplicación de diferentes tipos de control (desempeño global, control de ganancias y pérdidas e informes contables) evidencia la importancia de medir y corregir el plan estratégico con base en información clara, veraz y oportuna. Sin embargo, es necesario que el sector incorpore indicadores financieros y no financieros para evaluar de manera íntegra su gestión, lo que permite tener una visión global del desempeño, a través de la medición de los aspectos clave para el éxito de los agentes de aduanas.

Las múltiples herramientas de control de gestión aplicadas por los agentes de aduanas dan cuenta de su interés por analizar y evaluar sus resultados. Sin embargo, es posible que la gerencia esté duplicando sus esfuerzos producto de la falta de articulación entre las diferentes he- rramientas. Una vez integradas, es posible determinar la necesidad de generar otros indicadores no financieros, que sumados a los ya existen, han de proporcionar una visión global del desempeño.

\section{Referencias bibliográficas}

Álvarez Arango, Alberto (2005). Matemática Financiera. Tercera Edición. Bogotá, Editorial McGraw-Hill.

Asamblea Nacional de la República Bolivariana de Venezuela (2003). Control Cambiario. Gaceta Oficial Extraordinaria $N^{\circ} 37.625$, Decreto $N^{\circ} 2.302$, Venezuela.

Asamblea Nacional de la República Bolivariana de Venezuela (2008). Ley Orgánica de Aduanas. Gaceta Oficial Extraordinaria $\mathrm{N}^{\circ} 38.875$, Decreto $\mathrm{N}^{\circ}$ 5.879, Venezuela.

Bohórquez, Luz Esperanza (2011). Sistemas de control estratégico y organizacional. Críticas y desafíos. Revista Ciencias Estratégicas. Volumen 19, $N^{\circ} 26$, Medellín, Colombia, Universidad del Rosario, pp. 307-322.

Carrión Maroto, Juan (2007). Estrategia: de la Visión a la Acción. Segunda Edición. Madrid, Editorial ESIC.

Castaño, Germán (1998). Seminario de Teoría Administrativa: Control Estratégico. Universidad Nacional de Colombia. Disponible en: http://www.virtual.unal.edu.co/cursos/sedes/manizales/4010014/Contenidos/Capitulo4/Pages/4.15/415control_estrategico.htm. Fecha de consulta: 25-102011.

Chiavenato, Idalberto (2001). Administración: Proceso administrativo. Tercera Edición. México, Editorial Mc Graw-Hill Interamericana S.A.

Coopers \& Lybrand (1997). Los Nuevos Conceptos del Control Interno: Informe 
Coso. Madrid, Ediciones Díaz de Santos, S.A.

Ferrer, Thais y Pelekais, Cira de (2004). Tendencias Gerenciales y la Gestión Universitaria. Revista de Ciencias Sociales, Volumen $\mathrm{X}, \mathrm{N}^{\circ} 1$, Venezuela, Universidad del Zulia, pp 148-163.

Gitman, Lawrence (2003). Principios de Administración Financiera. México, Editorial Pearson Educación.

Harrison, Jeffrey y Caron, John (2009). Fundamentos de la Dirección Estratégica. España, segunda edición. Editorial Parafino, S.A.

Horngren, Charles; Datar, Srikant y Foster, George (2007). Contabilidad de Costos: Un enfoque gerencial. Décimo segunda edición. México, Editorial Pearson Educación.

Instituto Nacional de Estadística INE (2012). Sistema de consulta de estadísticas de comercio exterior (Importaciones y exportaciones). Venezuela. Disponible en: http://www.ine.gov. Ve/index.php?option=com_content\& view $=$ category\&id $=48 \&$ Itemid $=33 \#$. Fecha de consulta: 06-03-2012.

Kaplan, Robert y Norton, David (2009). EI Cuadro de Mando Integral (The Balanced Scorecard). Tercera Edición. España, Editorial Gestión 2000.

Kaplan, Robert; y Norton, David (1997). EI Cuadro de Mando Integral (The Balanced Scorecard). España, Ediciones Gestión 2000.

Martínez Pedrós, Daniel y Milla Gutiérrez, Artemio (2012). La elaboración del plan estratégico y su implantación a través del cuadro de mando integral. España, Ediciones Díaz de Santos.

Mintzberg, Henry; Quinn, James, Brian y Voyer, John (1997). El proceso estraté- gico. Conceptos, contextos y casos. México, Ediciones Pretince-Hall Hispanoamericana, S.A.

Münch, Lourdes y García, José (2005). Fundamentos de Administración. México, Editorial Trillas, S.A.

Muñoz, Carlos (2002). Auditoría en Sistemas Computacionales. México, Editorial Pearson Educación de México.

Nava, Marbelis; Urdaneta, Fátima y Casanova, Ángel (2008). Gerencia y productividad en sectores ganaderos de doble propósito. Revista Venezolana de Gerencia, Año 13, № 43, Venezuela, Centro de Estudios de la Empresa, Universidad del Zulia, pp. 468-491.

Navajo, Pablo (2009). Planificación estratégica en organizaciones no lucrativas. Guía participativa basada en valor. España, Ediciones Narcea, S.A.

Nieto, Raúl (2006). El cambio y el sentido de lo irracional: Incertidumbre, complejidad y caos. Primera Edición. Colombia, Editorial Pontificia Universidad Javeriana.

Porter, Lyman; Black, Stewart y Hitt, Michale (2006). Administración. México, Novena edición, Ediciones Pretince Hall Inc.

Quintero, Johana; Maza, Eliumat y Batista, Judeira (2010). Gerencia de Investigación y Desarrollo en Centros de Investigación de Universidades Públicas de Colombia. Revista Telos, Volumen 12 , № 3, Venezuela, Universidad Rafael Belloso Chacín, pp. 275-286.

\section{Entrevistas}

Arcaya, Germán. Presidente de la Asociación de Agentes Aduanales del estado Zulia (ASADAEZ); Maracaibo 05 de noviembre de 2007. 